\title{
Nutritional Evaluation of an Inter-Esterified Perilla Oil and Lard in Comparison with Butter and Margarine Based on the Survival of Stroke-Prone Spontaneously Hypertensive (SHRSP) Rats
}

\author{
Kenjiro Tatematsu, ${ }^{a}$ Natsuko Hirose,${ }^{a}$ Yuko Ichikawa, ${ }^{a}$ Yoichi Fujii, ${ }^{a}$ Akira Takami, ${ }^{b}$ \\ and Harumi Okuyama*, $a$ \\ ${ }^{a}$ Department of Preventive Nutraceutical Sciences, Faculty of Pharmaceutical Sciences, Nagoya City University, 3-1 Tanabedori, \\ Mizuhoku, Nagoya 467-8603, Japan and 'bamari Chemicals, Ltd., 1-4-29 Kunijima, Higashiyodogawa, Osaka 533-0024, Japan
}

(Received November 6, 2003; Accepted November 10, 2003)

\begin{abstract}
Some kinds of vegetable oil and a partially-hydrogenated oil shorten the survival of the stroke-prone spontaneously hypertensive (SHRSP) rats compared with perilla seed oil, soybean oil and lard. The n-3/n-6 ratio of constituent fatty acids, phytosterol content and other factors in these oils have been proposed to affect the survival of this strain. Here, we examined the safety of a fat produced by the inter-esterification of perilla oil and lard (Perilla-Lard) on the bases of the survival of SHRSP rats. The mean survival time decreased in the order of the butter, the Perilla-Lard, the lard, the margarine and the partially-hydrogenated soybean oil (Hyd.Soy) group. The correlations between survival time and cholesterol content or phytosterol content in the diet were analyzed, and the probable health benefits of the new margarine-type fats made of animal fats and oils with high n-3/n-6 ratios were discussed.
\end{abstract}

Key words — safety, butter, perilla oil, lard, strokeprone spontaneously hypertensive rat, survival

\section{INTRODUCTION}

The stroke-prone spontaneously hypertensive (SHRSP) rat strain, established from the SHR and Wistar/Kyoto strains, develops hypertension and cerebral hemorrhage, particularly when a salt solution is given as drinking water. These three strains develop phytosterolemia more easily than other rat strains possibly due to a mutation in a ABC transporter. ${ }^{1,2)}$ The addition of cholesterol to the diet prolongs the survival of this strain. ${ }^{3)}$ We found that

\footnotetext{
*To whom correspondence should be addressed: Department of Preventive Nutraceutical Sciences, Faculty of Pharmaceutical Sciences, Nagoya City University, 3-1 Tanabedori, Mizuhoku, Nagoya 467-8603, Japan. Tel. \& Fax: +81-52-8363427; E-mail: okuyamah@phar.nagoya-cu.ac.jp
}

canola oil, olive oil and some other oils shorten the survival of this strain by 15 to $40 \%$ depending on salt concentration as compared with soybean oil and perilla oil. ${ }^{4,5)}$

Partially-hydrogenated soybean oil (Hyd.Soy) has a survival-shortening activity comparable to that of canola oil ${ }^{4)}$ but lard is as safe as soybean oil. Fats and oils have different effects on survival of SHRSP rats that can not be accounted for by the difference in their fatty acid composition. Thus we assumed the presence of minor components in some vegetable oils (survival-shortening factors) to explain such differences. ${ }^{5)}$ Recently, phytosterols have been proposed to form a part of the survival-shortening factors. Along this line, olive oil is exceptional in that its phytosterol content is the lowest but survivalshortening activity is the highest among the oils examined. ${ }^{6}$ )

Efforts to identify the principle of survival-shortening factors have been continued, but it is possible to obtain a new type of fat without such survivalshortening activity by the inter-esterification of animal fat and perilla seed oil or flaxseed oil. The expected health benefits of such new types of margarine are as follows; their melting point can be modified by changing the ratio of fats and oils, and high n-3/n- 6 ratios can be maintained by choosing lowlinoleic acid fats and oils. Cholesterol in animal fats has long been considered as a detrimental factor for atherosclerosis-related diseases, but recent epidemiological studies revealed that serum cholesterol level negatively correlates with all-cause mortality and cancer mortality. ${ }^{7)}$ Therefore, cholesterol in animal fats is unlikely to be a risk factor for diseases in elderly. Moreover, saturated and monounsaturated fatty acids are not converted to eicosanoids, the over and unbalanced production of which is a major risk factor for these diseases. On the basis of this back- 
ground, we prepared margarine-type fats by the inter-esterification of animal fats and perilla seed oil, and compared the effect of an inter-esterification product (perilla oil and lard, Perilla-Lard) on the survival of SHRSP rats with those of butter, lard, a commercially available margarine and Hyd.Soy.

\section{MATERIALS AND METHODS}

Diets and Animals Test diets were prepared by mixing a basal conventional diet (CE-2; Central Laboratory of Experimental Animals, Clea Japan, Tokyo, Japan) with one type of fat at a $9: 1$ ratio. Butter (Y-brand) and margarine (N-brand) obtained from a local market were treated with hexane. The hexane layer was washed with water and the solvent was evaporated to obtain the fat fraction. Partially-hydrogenated soybean oil $\left(\mathrm{mp} ., 30^{\circ} \mathrm{C}\right.$, a product for human consumption) was purchased from Hamari Chemicals, Ltd. (Osaka, Japan). SHRSP rats were purchased from Seack Yoshitomi Co. (Fukuoka, Japan) and bred in our laboratory. Male rats from the same litter were divided randomly into different dietary groups and introduced to a test diet at 4 weeks old age. The rats were kept under specific pathogen-free conditions. Each of dietary groups comprised 12 animals and $0.25 \% \mathrm{NaCl}$ was given as drinking water. This experiment was approved by the Ethical Committee of the Graduate School of Pharmaceutical Sciences, Nagoya City University.

\section{Analysis of Sterols and Fatty Acids in Diets}

Sterols extracted from diets with hexane/chloroform (4:1) after saponification were treated with a trimethyl-silylating reagent (Tokyo Kasei Kogyo Co., Ltd., Tokyo, Japan), and the trimethylsilyl ethers were analyzed by gas-liquid chromatography (GLC). The fatty acids of the diets were analyzed as methylesters by GLC using heptadecanoic acid as an internal standard as described previously. ${ }^{8)}$

Inter-Esterification between Lard and Perilla Oil The inter-esterification reaction between lard and perilla oil $(1: 1)$ was performed with sodium methoxide as catalyst according to a conventional method used in cooking oil production. The degree of inter-esterification was monitored on the basis of the appearance of new molecular triacylglycerols using an HPLC system (LC-VP ver. 6.12 SP3) equipped with an evaporative light scattering detector (ELSD) detector (Shimadzu, Kyoto, Japan). $\left.{ }^{9}\right)$

Statistical Analyses — Data were presented as means \pm SEM. Statistical analysis of the survival data was performed by the Log-Rank and nonparametric Wilcoxon's signed rank tests. The computer program KyPlot ver. 2.0 (Kyence Inc., Tokyo, Japan) was used.

\section{RESULTS AND DISCUSSION}

The fatty acid and sterol compositions of the diets are shown in Table 1. Butter, lard and PerillaLard exhibited typical expected fatty acid compositions. From the fatty acid composition of margarine, it appears to be of the hard type composed of partially- hydrogenated vegetable oil(s) and untreated vegetable oil(s). The proportions of trans octadecenoic acid, $5.2 \%$ in the margarine and $19.2 \%$ in Hyd.Soy, can be a rough measure of the ratio of hydrogenated oil to untreated oil.

The mean survival time decreased in the order of butter, Perilla-Lard, lard, margarine and Hyd.Soy groups (Fig. 1). The mean survival time of the Hyd.Soy group was comparable to that of the canola oil group, and those of the butter and soybean oil groups were similar (data not shown).

The difference in the mean survival time between butter and Perilla-Lard groups was not significant ( $p=0.066$ in Log-Rank test and $p=0.051$ in the Wilcoxon's signed rank test), the mean survival time of Perilla-Lard group tended to be longer than that of lard group ( $p=0.053$ in the Log-Rank test and 0.033 in the Wilcoxon's signed rank test), the mean survival times of the butter and Perilla-Lard groups were significantly longer than that of the margarine group ( $p<0.002$ in both tests), and the difference between lard and margarine groups was significant $(p<0.02$ in both tests). The mean survival time of the Hyd.Soy group was significantly shorter than those of the butter, Perilla-Lard and lard groups ( $p=0.000$ in both tests), and also shorter than that of the margarine group ( $p<0.01$ in both tests).

Phytosterols, which are competitive inhibitors of cholesterol absorption in the intestine, have been shown to shorten the survival of SHRSP rats, but dietary cholesterols have been shown to prolong it. ${ }^{3,6}$ The contents of cholesterol and major phytosterols in the diets are shown in Table 1. The basal diet (CE2) contained cholesterol derived from fish meal and skimmed milk. In addition, butter, Perilla-Lard and lard contain cholesterol. The mean survival time correlated positively with cholesterol content in the diet and negatively with phytosterol content, except 
Table 1. Fatty Acid and Sterol Composition of Test Diet

\begin{tabular}{|c|c|c|c|c|c|}
\hline & Butter & Perilla-Lard & Lard & Margarine & Hyd. Soy \\
\hline \multicolumn{6}{|l|}{ Fatty acid (\%) } \\
\hline \multicolumn{6}{|l|}{ Saturated fatty acid } \\
\hline $12: 0$ & 1.7 & 0.0 & 0.0 & 0.1 & 0.0 \\
\hline $14: 0$ & 8.8 & 0.9 & 1.3 & 0.6 & 0.4 \\
\hline $15: 0$ & 1.0 & 0.1 & 0.1 & 0.1 & 0.0 \\
\hline $16: 0$ & 31.8 & 16.3 & 23.3 & 20.1 & 15.6 \\
\hline $18: 0$ & 8.5 & 6.1 & 10.6 & 4.2 & 5.9 \\
\hline $20: 0$ & 0.2 & 0.2 & 0.2 & 0.3 & 0.3 \\
\hline $22: 0$ & 0.1 & 0.0 & 0.0 & 0.2 & 0.3 \\
\hline \multicolumn{6}{|c|}{ Monounsaturated fatty acid } \\
\hline $14: 1$ & 0.7 & 0.1 & 0.0 & 0.0 & 0.0 \\
\hline $16: 1$ & 1.5 & 1.3 & 1.8 & 0.4 & 0.5 \\
\hline $18: 1 \mathrm{cis}$ & 23.3 & 28.3 & 36.4 & 32.5 & 28.2 \\
\hline $18: 1 \operatorname{trans}$ & 1.8 & 0.0 & 0.0 & 5.2 & 19.3 \\
\hline $20: 1$ & 0.3 & 0.7 & 0.8 & 0.6 & 0.5 \\
\hline $22: 1$ & 0.2 & 0.2 & 1.7 & 0.1 & 0.1 \\
\hline $24: 1$ & 0.1 & 0.0 & 1.8 & 0.1 & 0.0 \\
\hline \multicolumn{6}{|c|}{ n-6 Polyunsaturated fatty acid } \\
\hline $18: 2 n-6$ & 16.8 & 21.1 & 18.6 & 30.1 & 25.9 \\
\hline \multicolumn{6}{|c|}{ n-3 Polyunsaturated fatty acid } \\
\hline $18: 3 n-3$ & 1.4 & 23.1 & 1.1 & 3.2 & 1.3 \\
\hline $20: 5 n-3$ & 1.0 & 0.9 & 1.8 & 1.0 & 1.1 \\
\hline $22: 6 n-3$ & 0.6 & 0.5 & 0.5 & 0.8 & 0.6 \\
\hline \multicolumn{6}{|c|}{ Sterol $(\mathrm{mg} / 100 \mathrm{~g}$ diet $)$} \\
\hline Cholesterol & 75.5 & 62.3 & 65.2 & 53.2 & 54.1 \\
\hline Campesterol & 23.0 & 27.2 & 23.9 & 32.3 & 29.6 \\
\hline Stigmasterol & 8.4 & 11.0 & 9.3 & 12.6 & 14.0 \\
\hline$\beta$-Sitosterol & 47.1 & 71.0 & 49.9 & 66.0 & 82.0 \\
\hline Total phytosterol & 78.5 & 109.2 & 83.1 & 110.8 & 125.7 \\
\hline Total sterol & 153.9 & 171.5 & 148.3 & 164.1 & 179.8 \\
\hline
\end{tabular}

for the Perilla-Lard group; the phytosterol content in the Perilla-Lard diet was comparable to that in the margarine diet but the survival of the former was much longer than that of the latter $(p<0.02)$. This could be attributed to $\alpha$-linolenic acid-rich perilla oil, which is known to prolong the survival of SHRSP rats. ${ }^{8)}$ Similarly, high-linoleic and high-oleic types of safflower oil had similar phytosterol contents but their survival times were very different. ${ }^{4}{ }^{10)}$ Thus, phytosterol is a factor affecting the survival of SHRSP rats, but other factors are also involved.

The SHRSP, SHR and Wistar/Kyoto (WKY) rat strains are unique in that they develop phytosterolemia more easily than other rat strains. ${ }^{1,2)}$ Until now, the impact of the observed survival-shortening activity of some fats and oils on human nutrition remains inconclusive. ${ }^{11)}$ However, new types of fat (margarine) made of animal fats and perilla oil

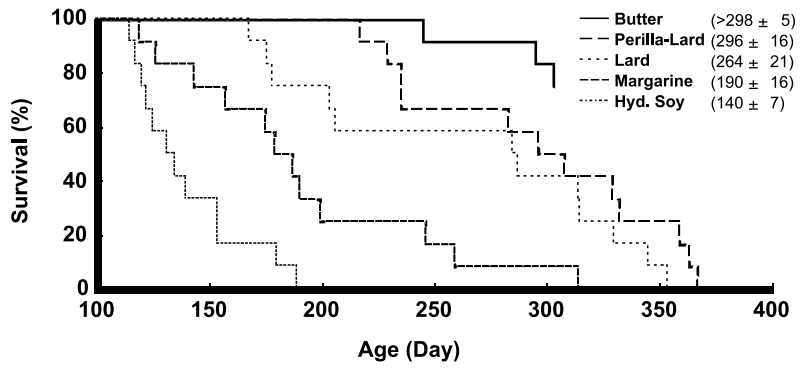

Fig. 1. Comparison of the Survival Curves and Mean Survival Days Shown in Parentheses

or flaxseed oil may be accepted particularly when the elevation of $n-3 / n-6$ ratio in foods is recommended and cholesterol intake is shown not to positively correlate with mortality from cardiovascular diseases. ${ }^{12)}$ 
Acknowledgements This work was supported in part by a Health and Labour Science Research Grant from the Ministry of Health, Labour and Welfare, Japan.

\section{REFERENCES}

1) Ikeda, I., Nakagiri, H., Sugano, M., Ohara, S., Hamada, T., Nonaka, M. and Imaizumi, K. (2001) Mechanisms of phytosterolemia in stroke-prone spontaneously hypertensive and WKY rats. Metabolism, 50, 1361-1368.

2) Scoggan, K. A., Gruber, H. and Lariviere, K. (2003) A missense mutation in the Abcg5 gene causes phytosterolemia in SHR, stroke-prone SHR, and WKY rats. J. Lipid Res., 44, 911-916.

3) Hamano, M., Mashiko, S., Onda, T., Tomita, I. and Tomita, T. (1995) Effects of cholesterol-diet on the incidence of stroke and life-span in malignant stroke prone spontaneously hypertensive rats. Jpn. Heart $J .$, 36, 511.

4) Miyazaki, M., Huang, M.-Z., Takemura, N., Watanabe, S. and Okuyama, H. (1998) Early mortality effect of partially hydrogenated vegetable oils in stroke-prone spontaneously hypertensive (SHRSP) rats. Nutr. Res., 18, 1049-1056.

5) Miyazaki, M., Watanabe, S., Oikawa, T., Morozumi, K., Fuzinami, T. and Okuyama, H. (1998) Free fatty acid fractions from some vegetable oils exhibit reduced survival time-shortening activity in strokeprone spontaneously hypertensive rats. Lipids, $\mathbf{3 3}$, 655-661.

6) Ratnayake, W. M. N., L'Abbe, M. R., Mueller, R.,
Hayward, S., Plouffe, L., Hollywood, R. and Trick, K. (2000) Vegetable oils in phytosterols make erythrocytes less deformable and shorten the life span of stroke-prone spontaneously hypertensive rats. J. Nutr., 130, 1166-1178.

7) Okuyama, H., Fujii, Y. and Ikemoto, A. (2000) n-6/ n-3 Ratio of dietary fatty acids rather than hypercholesterolemia as the major risk factor for atherosclerosis and coronary heart disease. J. Health Sci., 46, 157-177.

8) Shimokawa, T., Moriuchi, A., Hori, T., Saito, M., Naito, Y., Kabasawa, H., Nagae, Y., Matsubara, M. and Okuyama, H. (1988) Effect of dietary alphalinolenate/linoleate balance on mean survival time, incidence of stroke and blood pressure of spontaneously hypertensive rats. Life Sci., 43, 2067-2075.

9) Stolyhwo, A., Colin, H. and Guiochon, G. (1985) Analysis of triglycerides in oils and fats by liquid chromatography with the laser light scattering detector. Anal. Chem., 57, 1342-1354.

10) Huang, M.-Z., Naito, Y., Watanabe, S., Kobayashi, T., Nagatsu, A., Sakakibara, J. and Okuyama, H. (1997) Unusual effects of some vegetable oils on the survival time of stroke-prone spontaneously hypertensive rats. Lipids, 32, 745-751.

11) Sudhop, T., Gotwald, B. M. and von Bergmann, K. (2002) Serum sterols as a potential risk factor for coronary heart disease. Metabolism, 51, 1519-1521.

12) Hu, F. B., Stampfer, M. J., Rimm, E. B., Manson, J. E., Ascherio, A., Colditz, G. A., Rosner, B. A., Spiegelman, D., Speizer, F. E., Sacks, F. M., Hennekens, C. H. and Willett, W. C. (1999) A prospective study of egg consumption and risk of cardiovascular disease in men and women. JAMA, 281, 1387-1394. 